\title{
Supervivencia adulta y dinámica poblacional del lauchón orejudo Phyllotis darwini en Chile central
}

\author{
Adult survival and population dynamics in the leaf-eared mouse Phyllotis darwini \\ in central Chile
}

LAURENT CRESPIN ${ }^{1,2} \&$ MAURICIO LIMA ${ }^{1, *}$

\author{
${ }^{1}$ Center for Advanced Studies in Ecology \& Biodiversity, Departamento de Ecología, \\ Pontificia Universidad Católica de Chile, 193 Correo 22, Santiago CP 6513677, Chile \\ ${ }^{2}$ Centre de Biologie et de Gestion des Populations (UMR022); IRD Campus Internacional Agropolis de Baillarguet, \\ CS 30016, 34988 Montferrier-sur-Lez cedex, France; \\ * e-mail para correspondencia: mlima@bio.puc.cl
}

\begin{abstract}
RESUMEN
A nivel demográfico, los resultados clásicos de los modelos matriciales separan a las especies de tiempo generacional corto de las especies de tiempo generacional largo en cuanto a la importancia de la supervivencia adulta para la dinámica poblacional. Específicamente, la supervivencia adulta no debería contribuir de manera importante en la tasa de cambio poblacional de especies de tiempo generacional corto. Sin embargo, Yoccoz et al. (1998, Research Population Ecology 40: 107-121) propusieron que la supervivencia adulta sería el parámetro demográfico más importante para determinar la tasa de cambio poblacional en pequeños roedores cuando se toma en consideración una escala de tiempo mensual. Con el fin de poner a prueba esta hipótesis en este trabajo, utilizamos cinco años de datos de captura-marcaje-recaptura para estimar la supervivencia y la maduración de las hembras del lauchón orejudo, Phyllotis darwini, en una localidad de Chile central. El análisis mostró que las probabilidades de supervivencia disminuían con el promedio anual de la cantidad de lluvia y que las probabilidades de maduración disminuían con la densidad poblacional. Basados en las probabilidades de supervivencia y maduración, construimos un modelo matricial estacional para medir la importancia relativa de cada parámetro demográfico en el ciclo de vida de la especie a través de un análisis de perturbación. A fin de reflejar la variabilidad estacional del ambiente, dos estaciones fueron incorporadas en el modelo matricial: una estación de lluvia de cinco meses y una estación seca. Se observó que la supervivencia adulta era en efecto el parámetro demográfico con la elasticidad más fuerte. Por lo tanto, estos resultados apoyan la hipótesis de Yoccoz et al. (1998).
\end{abstract}

Palabras clave: maduración, supervivencia, modelo matricial, captura-marcaje-recaptura, Phyllotis.

\begin{abstract}
Classic results of matrix models predict that, in species with a long generation time, adult survival should be the demographic parameter driving population dynamics whereas, in species with a short generation time, adult survival should not be of such importance. Nonetheless, Yoccoz et al. (1998, Research Population Ecology 40: 107-121) hypothetized that, in small rodents, adult survival should be the demographic parameter driving the population growth rate if one considered a time scale of one month (instead of one year). As far as we know, this hypothesis has not yet been tested with empirical data. To test this hypothesis, we used five years of capture-mark-recapture data to estimate maturation and survival of a rodent, the leaf-eared mice, Phyllotis darwini, in a population of central Chile. This analysis revealed that the probabilities of survival decreased with the average rainfall by year and that the probabilities of maturation decreased with the abundance of the population. Using the probabilities of survival and maturation, we built up a seasonal matrix model and use perturbation analysis (elasticity) to be able to actually measure the relative importance of each demographic parameter to the population growth rate. Environmental seasonality was incorporated in the model by using a rainfall season, of five months long, and a dry season. Adult survival was indeed the demographic parameter with the highest elasticity. Such a result plainly supported thus the hypothesis of Yoccoz et al. (1998).
\end{abstract}

Key words: maturation, survival, matrix model, capture-mark-recapture, Phyllotis. 


\section{INTRODUCCIÓN}

La formalización del ciclo de vida a través de su traducción matemática, el modelo matricial, es una herramienta muy poderosa para conectar los procesos demográficos con la dinámica poblacional de diferentes tipos de organismos (Caswell 2001). En particular, los parámetros que se necesitan para armar un ciclo de vida son la supervivencia y la fecundidad. Los resultados clásicos de los análisis de perturbación de modelos matriciales básicos muestran que, en general, la dinámica poblacional de las especies de tiempo generacional largo está gobernada por la supervivencia adulta mientras que la dinámica poblacional de las especies de tiempo generacional corto depende sobre todo de los parámetros de fecundidad (Lebreton \& Clobert 1991). Para los mamíferos, los resultados empíricos generalmente apoyan este resultado aunque pueda existir mucha variación dentro de algunos grupos filogenéticos (Heppel et al. 2000). Por ejemplo, en los pequeños roedores, que tienen un tiempo de generación corto, Yoccoz et al. (1998) propusieron que la supervivencia adulta era el parámetro más influyente en la dinámica poblacional si uno considera, no la escala de tiempo anual, sino una escala de tiempo mensual, adecuada a este tipo de especies que raramente sobreviven más de un año. Una característica demográfica fundamental de los pequeños roedores es la existencia de varias generaciones de individuos durante el año, con diferentes estrategias de maduración, i.e. el pasaje del estado no reproductivo al estado reproductivo o adulto (Leirs et al. 1993, Kaneko et al. 1998, PrévôtJulliard et al. 1999). La mayoría de los trabajos recientes que utilizan datos de CapturaMarcaje-Recaptura, en pequeños roedores, han descartado el estudio directo de la maduración, prefiriendo emplear el método desarrollado por Julliard et al. (1999) que consiste en cortar las historias de captura individuales y tratar de una manera independiente los períodos durante los cuales un individuo es subadulto y adulto respectivamente (Lima et al. 1999, Crespin et al. 2002). En este sentido, el trabajo de PrévôtJulliard et al. (1999) constituye una excepción al utilizar un modelo multiestado (Hestbeck et al. 1994; Nichols et al. 1994) para dar cuenta explícitamente de la transición de subadulto a adulto a partir de las historias de captura completas. Un resultado interesante de dicho trabajo fue la demostración que la maduración de las hembras dependía negativamente de la cantidad de hembras reproductivas en una población del roedor Clethrionomys glareolus en Finlandia. Sin embargo, un aspecto importante a considerar es la ausencia de pruebas de bondad de ajuste para los modelos multiestado utilizados por Prévôt-Julliard et al. (1999). Estos tipos de pruebas son necesarias para evaluar los supuestos de estos modelos de estimación de parámetros demográficos a partir de datos de CMR y han sido desarrolladas recientemente por Pradel et al. (2003).

En síntesis, los procesos demográficos de maduración sexual, tan peculiares en la biología reproductiva de los pequeños roedores, han sido poco estudiados e incorporados como tales en modelos de dinámica poblacional (Lima et al. 2003). Por lo tanto, en este trabajo usamos modelos de captura-marcaje-recaptura multiestados, y también las pruebas de bondad de ajuste correspondientes para estimar los parámetros de supervivencia y de maduración de las hembras de una población de Phyllotis darwini Waterhouse (1837) en la localidad de Quebrada de la Plata, Chile central. Estas estimaciones empíricas se incluyeron en un modelo matricial estacional para evaluar la importancia relativa de cada proceso demográfico en la dinámica poblacional de esta especie.

\section{MATERIALES Y MÉTODOS}

Estudio de captura-marcaje-recaptura de Phyllotis darwini

El estudio de captura-marcaje-recaptura empezó en el mes de noviembre de 1998 pero descartamos las dos primeras sesiones de trampeo para analizar solo años completos (desde enero hasta diciembre). Entonces este trabajo está basado en 45 sesiones primarias de trampeo a lo largo de cinco años (1999-2003). Cada sesión de trampeo tuvo lugar durante cuatro días (es decir, con cuatro sesiones de trampeo secundarias). Por lo general, el tiempo entre dos sesiones primarias era de aproximadamente un mes pero en algunas pocas ocasiones fue diferente (mediana: 30 días 
rango: 27-123); entonces, estandarizamos todas las estimaciones de supervivencia a 28 días (i.e., la escala de tiempo de un mes sugerido por Yoccoz et al. en 1998 para modelar el ciclo de vida de los pequeños mamíferos).

La grilla de trampeo fue de 1,5 ha con 81 trampas Sherman en un matorral mediterráneo típico de la zona central de Chile $\left(33^{\circ} 28^{\prime} \mathrm{S}\right.$, $70^{\circ} 54^{\prime}$ O) (Fig. 1). Se utilizó avena como cebo. Cada individuo fue marcado con un crotal en la oreja, pesado, y se determinó su sexo y condición reproductiva.

Phyllotis darwini es un pequeño roedor (el promedio del peso de un individuo adulto es aproximadamente de $50 \mathrm{~g}$ ) con una distribución amplia en Chile, especialmente en los ambientes semiáridos y mediterráneos
(Meserve \& Le Boulengé 1987). Esta especie es herbívora-granívora. En el sitio de Quebrada de la Plata, la época reproductiva difiere de un año al otro, con un periodo de mayor intensidad que corresponde más o menos a la estación de lluvias. Sin embargo, se puede encontrar hembras preñadas o lactantes fuera de este periodo. De manera similar Meserve \& Le Boulengé (1987) reportaron un periodo reproductivo largo para Phyllotis darwini a pesar de los datos que corresponden a años secos. Por otro lado, hembras mantenidas en cautiverio en la región de Santiago se reprodujeron casi todo el año, lo que sugiere una conexión entre periodo reproductivo y disponibilidad de alimento. La dinámica poblacional de la especie presenta marcadas

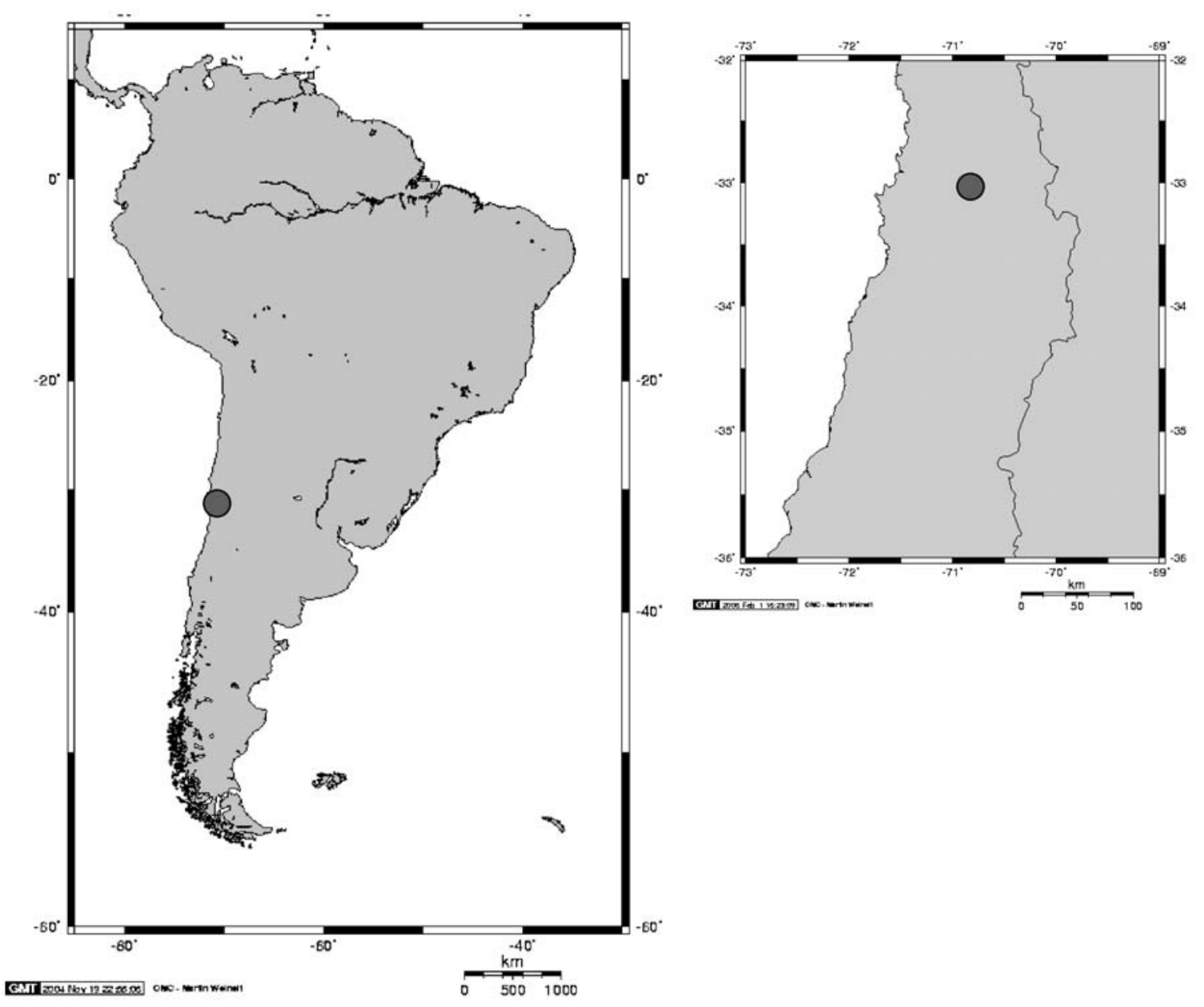

Fig. 1: El sitio de estudio, Quebrada de la Plata, está situado en la zona central de Chile $\left(33^{\circ} 28^{\prime} \mathrm{S}\right.$, $\left.70^{\circ} 54^{\prime} \mathrm{O}\right)$.

The study site, Quebrada de la Plata, is on the central region of Chile ( $\left.33^{\circ} 28^{\prime} \mathrm{S}, 70^{\circ} 54^{\prime} \mathrm{W}\right)$. 
fluctuaciones interanuales causadas por interacciones entre factores intrínsecos (densidad de los roedores con un efecto directo y retrasado) y varios factores externos como la lluvia o los depredadores (Lima \& Jaksic 1998a, 1998b, Lima \& Jaksic 1999, Lima et al. 1999, 2001).

\section{Modelos de captura-marcaje-recaptura}

Los modelos de captura-marcaje-recaptura permiten estimar los parámetros demográficos (supervivencia, reclutamiento y maduración) que actúan en una población natural, a partir de datos colectados con animales marcados, liberados y capturados varias veces. Las historias de captura de los individuos constituyen los datos básicos de aquellos modelos. Mediante algunas hipótesis básicas, que son puestas a prueba con las pruebas de bondad de ajuste, se puede estimar los parámetros demográficos con máxima verosimilitud (Lebreton et al. 1992). En este trabajo usamos un modelo multiestado con dos estados, subadulto $(<35 \mathrm{~g}$, Meserve \& Le Boulengé 1987) y adulto y con tres tipos de parámetros: probabilidades de supervivencia, de recaptura y de transición (es decir, en este caso, probabilidades de maduración). Para no aumentar desmedidamente el número de parámetros, hicimos el supuesto de que, una vez alcanzado el estado adulto, los individuos permanecían adultos (i.e., forzando la probabilidad de transición de adulto a subadulto a cero). Usamos el programa MARK para estimar los parámetros del modelo (White \& Burnham 1999). La calidad del ajuste a los datos del modelo más general fue comprobada según las recomendaciones de Pradel et al. (2003) con el programa UCARE versión 2.02 (Choquet et al. 2003), y para correr los modelos más generales usamos la versión 1.71 de MSURGE (Choquet et al. 2004). La selección de modelos fue llevado a cabo de acuerdo a la metodología expuesta en Lebreton et al. (1992), es decir, basado en el criterio de información de Akaike $\left(\mathrm{AIC}_{\mathrm{c}}\right)$. Se seleccionan los modelos de menor $\mathrm{AIC}_{\mathrm{c}}$ para hacer inferencias. Dos modelos con una diferencia en AICc de menos de dos son considerados como idénticos del punto de vista estadístico (Burnham \& Anderson 1998) y, en este caso, usamos el modelo que tiene el número menor de parámetros para inferencias, por el principio de parsimonia. La selección de modelos es un paso crucial para el análisis estadístico. Por lo general, en vez de probar todos los modelos posibles, se recomienda establecer un conjunto de modelos a priori, para no caer en la trampa del 'data-dredging' (Johnson \& Omland 2004). Por otro lado, el número de parámetros de un modelo es una función de la calidad/cantidad de los datos (Burnham \& Anderson 1998, Ginzburg \& Jensen 2004). Entonces, especialmente cuando uno trabaja con especies o poblaciones poco estudiadas, es difícil evaluar a priori la complejidad que es posible otorgar a los modelos y hay un riesgo de dejar afuera del conjunto de modelos uno o varios modelos con mayor poder explicativo (e.g., Dreitz et al. 2001 y las réplicas de Beissinger \& Snyder, y de Dreitz et al. 2002). En este trabajo, dado que era la primera vez que se analizaba la demografía de la población de Quebrada de la Plata y que había solo cinco años de datos, la selección de modelos fue llevada a cabo inspirándose en Grobois \& Tavecchia (2003). Partimos de un modelo que se ajustaba a los datos y de mayor complejidad. Esta complejidad fue elegida a priori e incluía variaciones estacionales y anuales en cada uno de los parámetros demográficos (ver más adelante el modelo 2). Modelamos primero las probabilidades de recaptura porque son las que menos aportan a la interpretación biológica (los así llamados parámetros 'ruidosos', Lebreton et al. 1992). Al reducir el número de parámetros en las probabilidades de recaptura, tratamos de encontrar un modelo aceptable, es decir, un modelo que no altere de una manera significativa el ajuste del modelo a los datos, quedando los otros parámetros modelados con la máxima complejidad elegida a priori. Después, utilizando este modelo aceptable para las probabilidades de recaptura, modelamos las probabilidades de supervivencia, tratando de reducir el número de parámetros y dejando las probabilidades de maduración con la máxima complejidad. En un tercer paso modelamos de la misma manera las probabilidades de maduración usando los modelos de recaptura y de supervivencia generados anteriormente. Finalmente, a partir de este modelo aceptable, intentamos reducir el número de parámetros para alcanzar un modelo desde el cual poner a prueba el efecto de las covariables. Elegimos a priori 
dos covariables importantes para caracterizar la variación anual de la supervivencia y maduración. Debido a que en un trabajo previo Lima et al. (2001) demostraron que la densidad y la lluvia eran dos variables cruciales para entender la dinámica poblacional de una población de $P$. darwini, utilizamos las mismas dos covariables. Las estimaciones de abundancia se calcularon en el periodo de máxima abundancia de los roedores (enerofebrero). Utilizamos estimaciones de abundancia basadas en el modelo con heterogeneidad de captura de poblaciones cerradas para los años 1999 a 2002 (modelo M(h), ver Otis et al. 1978 para la teoría y Leirs 1994 para un ejemplo). En 2003, año de baja densidad, por falta de capturas, no fue posible calcular un estimación de la abundancia mediante este método; entonces, siguiendo a Yoccoz \& Mesnager (1998), usamos el mínimo número de individuos vivos como estimación de la abundancia ('MNKA', Krebs 1966). Cabe señalar que las abundancias estimadas por los dos métodos eran similares para los años 1999-2002. Para enfatizar las diferencias ambientales entre años, usamos como variable anual el promedio de lluvia caído en cada mes ya que varios autores describieron la lluvia como un factor clave para la dinámica poblacional de $P$. darwini y especialmente asociada a las "ratadas" que ocurren en las zonas semiáridas del centro-norte de Chile (ver referencias en Meserve \& Le Boulengé 1987). Los datos de lluvia fueron proporcionados por el centro de información de recursos hídricos y colectados en una estación situada a unos $4 \mathrm{~km}$ del sitio de estudio (terraza oficinas centrales Dirección General de Aguas; $33^{\circ} 26^{\prime} \mathrm{S}, 70^{\circ} 39^{\prime} \mathrm{O}$ ). La cantidad de lluvia varió bastante durante el periodo, con un promedio mensual por año de $30,6 \mathrm{~mm}$ en 1999 , de 41,6 en 2000, de 29,4 en 2001, de 51,6 en 2002 y de 19,0 en 2003. Esperamos una relación positiva entre la supervivencia y/o la maduración y el promedio de lluvia. De acuerdo con las recomendaciones de White \& Burnham (1999), estas dos covariables anuales fueron estandarizadas.

Para modelar la estacionalidad, dado que la cantidad de lluvia es un factor crucial para explicar la dinámica de población de $P$. darwini (Lima \& Jaksic 1999, Lima et al. 1999, 2002), usamos dos estaciones, una estación seca y una estación lluviosa. Para facilitar las comparaciones con los estudios anteriores (e.g., Meserve \& Le Boulengé 1987), usamos una estación lluviosa desde mayo hasta septiembre incluido, durante la cual cayó, en promedio, el $80 \%$ de las precipitaciones anuales en el periodo 1995-2004. Esperamos una supervivencia y una maduración mayor en estación de lluvia.

\section{Modelo matricial}

Según las recomendaciones de Yoccoz et al. (1998), para modelar el ciclo de vida de los pequeños roedores utilizamos un modelo estacional, con una estación de lluvia de cinco meses y una estación seca de siete meses y con un paso de tiempo de un mes. El modelo es restringido a las hembras, con dos grupos, subadultas y adultas. En cada mes, una fracción de las hembras subadultas se vuelve adulta y la otra permanece subadulta. Solo las hembras adultas se reproducen. Dado que contamos con solo cinco años de datos, no intentamos modelar la variabilidad anual de la tasa de multiplicación poblacional y usamos un modelo matricial estacional con coeficientes constantes. Con este tipo de modelo, es posible calcular la contribución de cada parámetro mensual a la tasa de multiplicación asintótica de la población $(\lambda)$ en términos de su elasticidad (análisis de perturbación ver Caswel 2001, capitulo 13). De acuerdo con la hipótesis formulada por Yoccoz et al. (1998), se espera que la supervivencia adulta tenga una mayor elasticidad.

Como valores de los parámetros demográficos del modelo matricial, usamos el promedio de las probabilidades de supervivencia y maduración obtenidas por el análisis de CMR, es decir, para cada estación, un promedio sobre los cinco años del estudio. Para $P$. darwini, Meserve \& Le Boulengé (1987) describieron un tamaño de camada de dos a seis crías con un promedio de 5,2 y dos a tres camadas en la estación reproductiva. Arana et al. (2002) reportaron tamaños de camada similares para dos especies peruanas $P$. andium y $P$. amicus (uno a cuatro y uno a tres respectivamente). En general los parámetros de números de crías por camada y de números de camadas son poco variables en roedores (Yoccoz et al. 1998), por lo tanto en este trabajo tomamos un valor de 2,6 crías por mes en la estación de lluvia, que es la estación donde tiene lugar la reproducción. En la 
estación seca, donde se produce poca reproducción, usamos un valor de 1. Sin embargo, comprobamos que los resultados obtenidos sobre las sensibilidades de la supervivencia adulta eran cualitativamente los mismos con un valor de $0,75,0,5$ o de 0,25 crías por mes en la estación seca (datos no publicados). La supervivencia juvenil es, por lo general, un parámetro poco conocido en vertebrados por las dificultades que hay en marcar y capturar juveniles (Yoccoz et al. 1998, Nur \& Sydeman 1999) y no existe algún estudio de este parámetro por $P$. darwini. Entonces, se supone que, durante su primer mes de vida, la probabilidad de supervivencia de un juvenil es igual a la probabilidad de supervivencia de la madre (Lima et al. 2003).

\section{RESULTADOS}

\section{Abundancia}

Las abundancias con sus intervalos de confianza de $95 \%$ en paréntesis son: en $199915(13 ; 27)$, en $20009(9 ; 23)$, en $200137(30 ; 56)$ y en 2002 5 (2; 11). En el 2003, año de baja densidad, el mínimo número de individuos vivos era de 2 .

\section{Estudio de captura-marcaje-recaptura}

Se registraron 129 capturas únicas representando 260 individuos en el conjunto de datos. De ellos, había 114 individuos subadultos (con 359 capturas) y 22 adultos (con 126 capturas), 35 individuos fueron capturados como subadulto y adulto. Ninguna de las pruebas de bondad de ajuste resultó significativa, lo que sugiere que el modelo más general se ajusta satisfactoriamente a los datos $\left(3 \mathrm{SR} \chi^{2}=22,85\right.$ g.1. $=30 ; 3 \mathrm{GSM} \chi^{2}$ $=7,31$, g.1. $=30$; ITEC $\chi^{2}=17,04$, g.1. $=13$; LTEC $\chi^{2}=1$, g.l. $=3$; prueba de razón de verosimilitud máxima $=8,70$, g.l. $=88$; todos los valores de $\mathrm{P} \geq 0,2)$. A continuación realizamos una selección de modelos a partir del modelo general que cuenta con 218 parámetros (modelo $1 \mathrm{AIC}_{\mathrm{c}}=1649,47$; Tabla 1 ).

En primer lugar, dado el gran número de parámetros en el modelo más general y la cantidad de datos, reducimos la variabilidad temporal en variabilidad estacional, anual e interacciones; el modelo 2 , con solo 50 parámetros, tiene un $\mathrm{AIC}_{\mathrm{c}}$ mucho más bajo que el modelo 1 (ver Tabla 1). Desde este modelo, al simplificar las probabilidades de recaptura, fue posible eliminar las interacciones, quedando un modelo puramente aditivo con el estadio, el año

\section{TABLA 1}

Selección de modelos desde el modelo más general que se ajustaba a los datos de una manera satisfactoria; DEV representa la desvianza, np el número de parámetros identificables y $\mathrm{AIC}_{\mathrm{c}}$ el criterio de información de Akaike. La variabilidad temporal total es representada por t, la variabilidad anual por an y la variabilidad estacional por es. La variable del estado (madura/no madura) esta representada por st. El mejor modelo de este paso está descrito en negrita

Model selection from the more general model which fitted the data. Best model at this stage of model selection is shown in bold. DEV: deviance; np: number of estimable parameters; $\mathrm{AIC}_{\mathrm{c}}$ : Akaike's Information Criterion; t: total temporal variation; an: between-years variation; es: seasonal variation; st: state variation (subadult and adult individuals)

\begin{tabular}{lllllll}
\hline Modelo & Supervivencia & Recaptura & Maduración & DEV & np & AIC \\
\hline 1 & st*t & st*t & t & 346,29 & 218 & 1649,47 \\
2 & st*an*es & st+an*es & an*es & 519,2 & 50 & 1085,73 \\
3 & st*an*es & st+an*es & an*es & 535,92 & 41 & 1080,19 \\
$\mathbf{4}$ & st*an*es & st+an+es & an*es & $\mathbf{5 4 0 , 2 2}$ & $\mathbf{3 7}$ & $\mathbf{1 0 7 4 , 8 7}$ \\
5 & st*an*es & st+an & an*es & 544,22 & 36 & 1076,50 \\
6 & st*an*es & st+es & an*es & 565,32 & 33 & 1090,54 \\
7 & st*an*es & an+es & an*es & 540,80 & 36 & 1073,08 \\
8 & st*an*es & Es & an*es & 565,50 & 32 & 1088,40 \\
9 & st*an*es & An & an*es & 544,43 & 35 & 1074,35 \\
10 & st*an*es & St & an*es & 568,37 & 32 & 1091,26 \\
11 & st*an*es & an*es & 568,43 & 31 & \\
\hline
\end{tabular}


y la estación (modelo 4; $\mathrm{AIC}_{\mathrm{c}}=1074,87$ ). Por otra parte, el modelo 7, con año y estación, y el modelo 9, con solo año, tienen un $\mathrm{AIC}_{\mathrm{c}}$ un poco menor al modelo 4 (respectivamente $\Delta \mathrm{AIC}_{\mathrm{c}}=$ $1,79$ y 0,52$)$. Sin embargo, para no aumentar el riesgo de cometer un error de tipo I en esta etapa de la selección de modelos, utilizamos el modelo 4 como base para seguir en la selección de modelos (Pradel et al. 1990).

Remover las interacciones en las probabilidades de supervivencia provocó una baja del $\mathrm{AIC}_{\mathrm{c}}$ de más de 17 (modelo 13, Tabla 2). Otros dos modelos (14 y 15) presentaron $\mathrm{AIC}_{\mathrm{c}}$ similares al modelo 13, aunque un poco mayor, con solo estación y año o estado y año (respectivamente $\Delta \mathrm{AIC}_{\mathrm{c}}=0,51$ y 1,96 ). Por la misma razón que anteriormente, elegimos entonces al modelo 13 para seguir en la selección de modelos. Desde el modelo 13, era posible remover las interacciones de las probabilidades de maduración sin provocar una alza significativa del $\mathrm{AIC}_{\mathrm{c}}\left(\Delta \mathrm{AIC}_{\mathrm{c}}=1,83\right.$ modelo 21, Tabla 2). Dado que los otros modelos con menos parámetros tenían un $\mathrm{AIC}_{\mathrm{c}}$ bastante mayor (un aumento mínimo de más de 2,5) usamos el modelo 21 para seguir en la selección de modelos y hallar un modelo aceptable desde el cual poner a prueba las covariables.

Reducimos el número de parámetros del modelo 21 al modelo 26, eliminando la variable estado (modelo 21 versus modelos 25 y 26, Tabla 3). No fue posible seguir simplificando el modelo 26; en cambio, reintroducir las interacciones en las probabilidades de maduración condujo a un mejor modelo (modelo 35, $\Delta \mathrm{AIC}_{\mathrm{c}}=1,97$ ).

Desde el modelo 35, se realizó la puesta a prueba de las covariables en las probabilidades de supervivencia y de maduración. Al incluir la cantidad de lluvia en las probabilidades de supervivencia, se produjo una baja significativa del $\mathrm{AIC}_{\mathrm{c}}$ (modelo 35 versus modelo $39 \Delta \mathrm{AIC}_{\mathrm{c}}=$ 2,4 , ver Tabla 4). No había ninguna evidencia que añadir la lluvia con un retraso de un año mejoraba el $\mathrm{AIC}_{\mathrm{c}}$ (modelos 39 versus 41 y 37 versus 38 ). La abundancia, aunque disminuyó el $\mathrm{AIC}_{\mathrm{c}}$ comparado con el modelo 35, no causó una baja significativa $\left(\Delta \mathrm{AIC}_{\mathrm{c}}=1,31\right)$. En cuanto a las probabilidades de maduración, incluir la cantidad de lluvia y la abundancia reducía levemente el $\mathrm{AIC}_{\mathrm{c}}$ (modelo 39 versus $42 \Delta \mathrm{AIC}_{\mathrm{c}}=0,78$ ). $\mathrm{Sin}$ embargo, era posible remover las interacciones $\mathrm{y}$ la cantidad de lluvia de las probabilidades de maduración sin causar una disminución significativa del ajuste del modelo (modelos 42 versus $\left.45 \Delta \mathrm{AIC}_{\mathrm{c}}=1,13\right)$. Remover cualquier parámetro del modelo 45 se traducía por un aumento significativo del $\mathrm{AIC}_{\mathrm{c}}$ (mínimo aumento: 2,67). De acuerdo con el principio de parsimonia, elegimos el modelo 45 como el mejor modelo.

TABLA 2

Selección de modelos en las probabilidades de supervivencia y maduración (misma leyenda que Tabla 1)

Model selection for survival and maturation probabilities (same legend as Table 1)

\begin{tabular}{|c|c|c|c|c|c|c|}
\hline Modelo & Supervivencia & Recaptura & Maduración & DEV & $\mathrm{np}$ & $\mathrm{AIC}_{\mathrm{c}}$ \\
\hline 4 & st*an*es & st+an+es & an*es & 540,22 & 37 & 1074,87 \\
\hline 12 & st+an*es & st+an+es & an*es & 550,77 & 28 & 1064,46 \\
\hline 13 & st+an+es & st+an+es & an*es & 552,96 & 24 & 1057,62 \\
\hline 14 & an+es & st+an+es & an*es & 555,71 & 23 & 1058,13 \\
\hline 15 & st+es & st+an+es & an*es & 563,79 & 20 & 1059,58 \\
\hline 16 & $\mathrm{st}+\mathrm{an}$ & st+an+es & an*es & 567,87 & 23 & 1070,29 \\
\hline 17 & es & st+an+es & an*es & 573,67 & 19 & 1067,27 \\
\hline 18 & an & st+an+es & an*es & 571,63 & 22 & 1071,83 \\
\hline 19 & st & st+an+es & an*es & 578,05 & 19 & 1071,65 \\
\hline 20 & . & st+an+es & an*es & 588,97 & 18 & 1080,38 \\
\hline 21 & st+an+es & st+an+es & an+es & 563,67 & 20 & 1059,45 \\
\hline 22 & st+an+es & st+an+es & es & 583,86 & 16 & 1070,94 \\
\hline 23 & $\mathrm{st}+\mathrm{an}+\mathrm{es}$ & st+an+es & an & 568,44 & 19 & 1062,04 \\
\hline 24 & st+an+es & st+an+es & . & 598,18 & 15 & 1083,12 \\
\hline
\end{tabular}


TABLA 3

Selección de modelos para hallar un modelo aceptable para poner a prueba las covariables elegidas (leyenda como en Tabla 1)

Model selection for reaching an acceptable model to test the covariates (legend as in Table 1)

\begin{tabular}{lllllll}
\hline Modelo & Supervivencia & Recaptura & Maduración & DEV & np & AIC $_{c}$ \\
\hline 21 & st+an+es & st+an+es & an+es & 563,67 & 20 & 1059,45 \\
25 & an+es & st+an+es & an+es & 565,06 & 19 & 1058,66 \\
26 & an+es & an+es & an+es & 567,23 & 18 & 1058,65 \\
27 & an+es & an & an+es & 570,62 & 17 & 1059,86 \\
28 & an+es & Es & an+es & 591,11 & 14 & 1073,90 \\
29 & es & an+es & an+es & 584,82 & 14 & 1067,61 \\
30 & an & an+es & an+es & 582,97 & 17 & 1072,21 \\
31 & an+es & st+an+es & an+es & 566,38 & 19 & 1059,98 \\
32 & es & st+an+es & an+es & 584,35 & 15 & 1069,29 \\
33 & st+an+es & an & an+es & 568,33 & 18 & 1059,75 \\
34 & st+es & an+es & an+es & 575,67 & 15 & 1060,60 \\
$\mathbf{3 5}$ & an+es & an+es & an*es & $\mathbf{5 5 6 , 4 8}$ & $\mathbf{2 2}$ & $\mathbf{1 0 5 6 , 6 8}$ \\
\hline
\end{tabular}

TABLA 4

Selección de modelos con covariables; abun es la abundancia de Phyllotis darwini y abunr es la abundancia con un retraso de 1 año; lluv representa el promedio de lluvia caída cada mes y lluvr la misma variable con un retraso de 1 año

Model selection with covariates; abun: maximal annual abundance of Phyllotis darwini; abunr: covariate abun with one year lag; lluv: mean monthly rainfall over one year; llvr: covariate lluv with one year lag

\begin{tabular}{lllllll}
\hline Modelo & Supervivencia & Recaptura & Maduración & DEV & np & AIC \\
\hline 35 & an+es & an+es & an*es & 556,48 & 22 & 1056,68 \\
36 & es+lluv+abun+abun,1luv & an+es & an*es & 557,84 & 21 & 1055,83 \\
37 & es+lluv+abun & an+es & an*es & 558,93 & 20 & 1054,71 \\
38 & es+lluv+abun+abunr & an+es & an*es & 556,48 & 21 & 1056,68 \\
39 & es+lluv & an+es & an*es & 560,68 & 19 & 1054,28 \\
40 & es+abun & an+es & an*es & 561,77 & 19 & 1055,37 \\
41 & es+lluv+lluvr & an+es & an*es & 558,49 & 20 & 1054,28 \\
42 & es+lluv & an+es & lluv+es*abun & 570,70 & 14 & 1053,50 \\
43 & es+lluv & an+es & lluv+es+abun & 573,04 & 13 & 1053,71 \\
44 & es+lluv & an+es & es,abun & 574,00 & 13 & 1054,67 \\
$\mathbf{4 5}$ & es+lluv & an+es & es+abun & $\mathbf{5 7 6 , 0 8}$ & $\mathbf{1 2}$ & $\mathbf{1 0 5 4 , 6 3}$ \\
46 & es+lluv & an+es & Es & 592,24 & 11 & 1068,67 \\
47 & es+lluv & an+es & es+lluv & 578,53 & 12 & 1057,07 \\
\hline
\end{tabular}

El mejor modelo enfatiza que la supervivencia baja con el promedio de la lluvia en contra de nuestras expectativas (en la escala logit: $\beta$ (lluvia) $=-0,75$ con un intervalo de confianza de $95 \%$ de $[-1,16 ;-0,34])$ y que la maduración disminuye con la abundancia de los roedores $(\beta$ (abundancia) $=-0,95[-1,48 ;-0,41])$.
Además, la supervivencia y la maduración son más altas en la estación de lluvia como esperado (respectivamente $\beta$ (estación de lluvia) $=0,85$ $[0,41 ; 1,28]$ y $1.17[0,24 ; 2,10])$. En términos de probabilidades, la diferencia en supervivencia o en maduración entre la estación de lluvia y la estación seca es fuerte (Fig. 2). Cabe añadir que 
las estimaciones son también bastante diferentes de un año al otro (e.g., las probabilidades de maduración en 2001 comparado con el 2003). No existieron diferencias significativas entre las probabilidades de supervivencia de subadultas y adultas.

\section{Modelo matricial}

Como valores de los parámetros demográficos del modelo, utilizamos, para cada estación, el promedio de las cinco probabilidades de maduración y de supervivencia obtenidas desde el modelo 45 (maduración durante la estación lluviosa $=0,298$; estación seca $=0,123$; supervivencia durante la estación lluviosa $=$ 0,806; estación seca $=0,648)$. El modelo anual proyectaba una tasa de multiplicación de 1,60. Las elasticidades de los parámetros de las matrices mensuales a la tasa de multiplicación anual eran por lo general bastante similares entre los meses de una misma estación y diferentes de una estación a la otra (ver Tabla 5). Sin embargo, la supervivencia adulta era el parámetro con la mayor elasticidad en todos los meses.
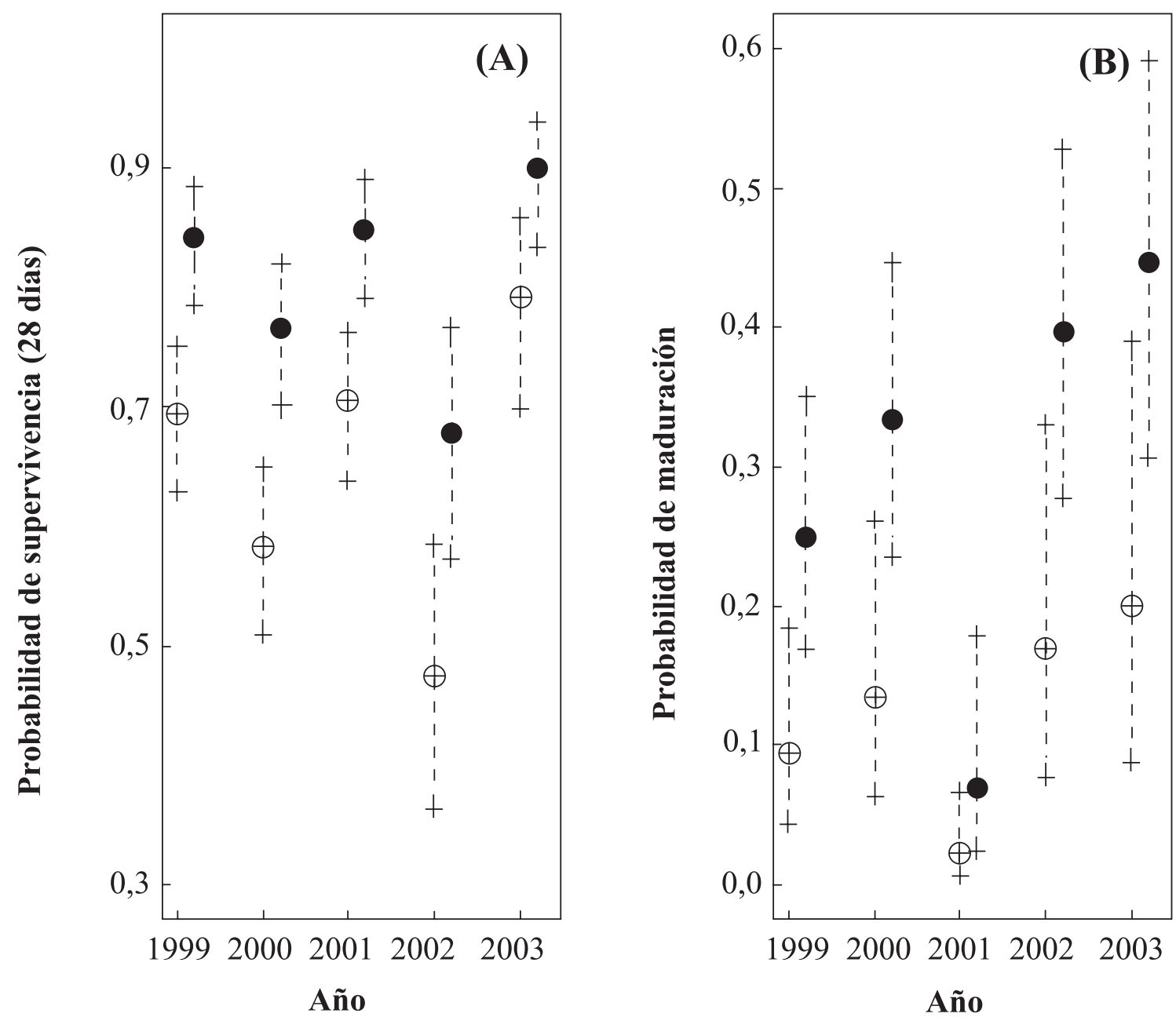

Fig. 2: (A) Variación de las probabilidades de supervivencia estandarizadas a 28 días. Los círculos representan las probabilidades durante la estación seca y los discos durante la estación lluviosa. Las líneas discontinuadas dan el intervalo de confianza a $95 \%$. (B) Variación de las probabilidades de maduración. La leyenda es la misma que en (A).

(A) Temporal variation of survival probabilities (estimates standardised to $28 \mathrm{~d}$ ). Open circles represent the estimates in the dry season and filled circles those in the rainy season. Dashed bars represent the 95\% confidence interval. (B) Temporal variation of maturation probabilities. Legend as in (A). 


\section{TABLA 5}

Elasticidades típicas de la tasa de multiplicación anual a los parámetros demográficos de las matrices mensuales (con el número de camada por mes fuera de la estación reproductiva igual a uno), $\mathrm{F}$ es la elasticidad de la tasa de multiplicación anual a la transición adulto a subadulto, es decir, la fecundidad, $\mathrm{S}$ es la elasticidad a la transición adulto a adulto, es decir, la supervivencia adulta, $\mathrm{N}$ es la elasticidad a la transición de subadulto a subadulto, $\mathrm{M}$ es la elasticidad a la transición subadulto a adulto, i.e., los procesos de maduración

Typical elasticities of annual population growth rate to demographic parameters from the monthly matrix model (number of litter per month taken as one in the non breeding season), $\mathrm{F}$ is the elasticity of annual population growth rate to the transition adult to subadult, i.e., fecundity, $\mathrm{S}$ is the elasticity to the transition adult to adult, i.e., adult survival, $\mathrm{N}$ is the elasticity to the transition subadult to subadult, $\mathrm{M}$ is the elasticity to the transition subadult to adult, i.e., maturation

\begin{tabular}{llllll}
\hline & \multicolumn{2}{c}{ Estación lluviosa } & & \multicolumn{2}{c}{ Estación seca } \\
\cline { 2 - 3 } \cline { 5 - 6 } Subadulto & Adulto & & Subadulto & Adulto \\
\hline Subadulto & N: 0,164 & F: 0,253 & & N: 0,290 & F: 0,130 \\
Adulto & M: 0,243 & S: 0,340 & & M: 0,140 & S: 0,440 \\
\hline
\end{tabular}

\section{DISCUSIÓN}

En cuanto al modelo matricial, nuestros resultados apoyan la hipótesis de Yoccoz et al. (1998). Con una escala de tiempo mensual, el parámetro demográfico que más influye en la tasa de multiplicación de $P$. darwini es la supervivencia adulta. Como es la primera vez, por lo que sabemos, que se pone a prueba esta hipótesis, ahora se necesitan otros ejemplos para saber si esta relación es una generalidad al nivel de los pequeños roedores. También sería interesante comprobar si esta relación se mantiene cuando uno modela al mismo tiempo la variabilidad estacional y anual.

En cuanto al estudio de CMR, la supervivencia y la maduración son más altas en la estación de lluvia, como se esperaba. En cuanto a la estacionalidad, el mejor modelo establece, para los procesos de maduración, que la mayoría de los individuos maduran, del estado subadulto al adulto, durante la estación lluviosa antes que empiece la estación seca. Sin embargo, algunos individuos maduran también en la estación seca por lo que las probabilidades de maduración, si bien son mucho más bajas que durante la estación lluviosa, no son iguales a cero (Fig. 2). Meserve \& Le Boulengé (1987) reportaron también que algunos machos probablemente nacidos en agosto o septiembre, maduraron en noviembre para otra población de $P$. darwini (Parque Nacional Fray Jorge, unos 400 km más al norte). Estas observaciones sugieren que, como otra especie africana de roedor de ambiente semiárido, Mastomys natalensis, la lluvia tiene un papel importante para desencadenar la reproducción. Para $M$. natalensis, experimentos de laboratorio demostraron que una dieta con brotes de nuevas plantas del año estimulaba la reproducción (Leirs et al. 1994). Por otro lado, se ha reportado que algunos componentes químicos presentes en los brotes de las nuevas plantas del año provocan un aumento del peso de los ovarios para $M$. natalensis y constituyen un estímulo reproductivo para Microtus (Korn 1989, Linn 1991). Entonces el retraso de algunas semanas entre el principio de las lluvias y la reproducción probablemente se explicaría porque no todos los individuos tienen el mismo acceso a los brotes de las nuevas plantas del año (Leirs et al. 1994).

Por otro lado, la supervivencia adulta es también más alta durante la estación de lluvia, lo cual concuerda con resultados anteriores sobre otras poblaciones de la misma especie. Primero, Meserve \& Boulengé (1987) describieron una cumbre en la supervivencia en invierno para $P$. darwini en la población del Parque Nacional de Fray Jorge. Segundo, Lima et al. (1999) señalan que otoño-invierno sería el período de mayor supervivencia tanto para los subadultos como para los adultos en la población de $P$. darwini de Aucó (unos 100 km más al sur). Esta estacionalidad está en sintonía también con otros estudios que, para especies de roedores del hemisferio Norte, describieron una supervivencia más alta en invierno (Ostfeld \& Canham 1995, Yoccoz \& Mesnager 1998, Sinclair \& Lochmiller 2000, Crespin et al. 2002). Esta observación podría ser fortuita o una consecuencia de la baja, en invierno, de los costos asociados con la reproducción. 

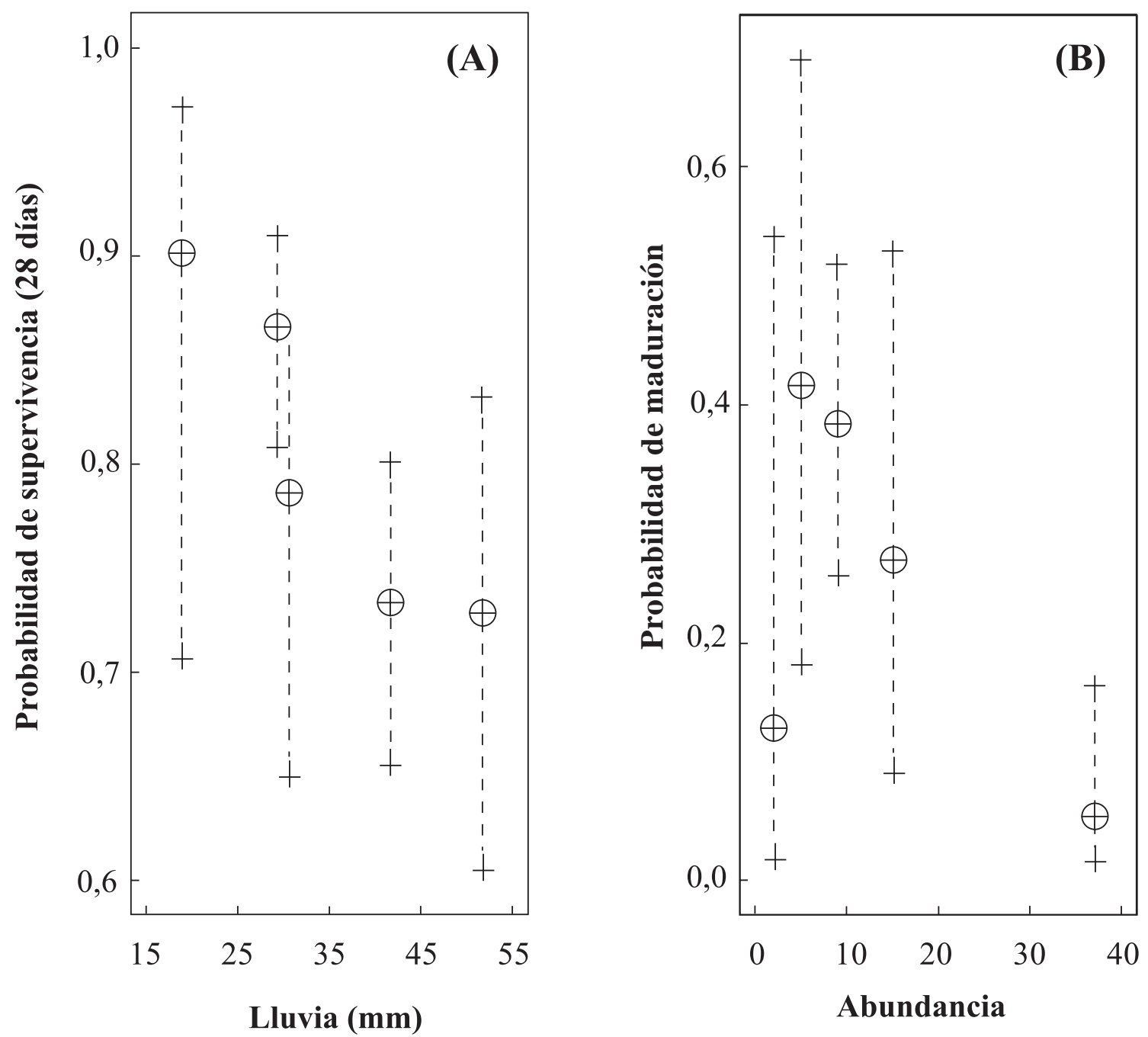

Fig. 3: Probabilidades de supervivencia y maduración en función de las covariables. Las estimaciones de los parámetros demográficos provienen de un modelo con variaciones estaciónales y anuales aditivas sin ninguna covariable (modelo 38, Tabla 2). Las líneas discontinuadas dan el intervalo de confianza a $95 \%$. (A) Supervivencia y lluvia. (B) Maduración y abundancia de Phyllotis darwini.

Survival and maturation probabilities plotted against values of covariates. Point estimates come from a model without any covariates (model 38, Table 2). Dashed bars represent the $95 \%$ confidence interval. (A) Survival versus rainfall. (B) Maturation versus abundance of Phyllotis darwini.

$\mathrm{Si}$ bien un periodo de cinco años puede parecer poco para entender la variabilidad interanual demográfica de un pequeño roedor, estos cinco años presentaron una variabilidad fuerte en relación con la abundancia de roedores con años de baja y alta densidad y una importante variabilidad en cuanto a los niveles de precipitaciones. Por otro lado, las relaciones encontradas con la abundancia poblacional y la cantidad de lluvia son robustas. Primero las estimaciones de supervivencia y maduración de un modelo con solo variaciones aditivas estaciónales y anuales (es decir, el modelo 26 que no contiene ninguna covariable, ver Tabla 3 ) muestran las mismas tendencias con la lluvia y la abundancia que las descritas por el mejor modelo (Fig. 3). Segundo, al remover en turno un año cada vez, la relación entre el parámetro demográfico y la covariable se conserva igual con una pendiente negativa en la escala logit en los cinco casos posibles. La relación entre 
la abundancia poblacional y la probabilidad de maduración parece menos clara a causa de una estimación pobre de la supervivencia en 2003 debida a las pocas recapturas de este año (reflejo de la abundancia mínima alcanzado en los cinco años como hemos mencionado anteriormente). Si bien la relación entre la supervivencia y la lluvia fue negativa, en contra de nuestras expectativas, Julliard et al. (1999) demostraron también una relación negativa entre la lluvia y la supervivencia en un roedor africano, que habita ambientes semiáridos, $M$. natalensis, aunque a una escala diferente (ellos usaron la cantidad de lluvia caída en el mes). En el caso de $P$. darwini, el efecto negativo de la lluvia se puede relacionar con las bajas de las temperaturas asociadas con las lluvias fuertes en el otoño e invierno o también por el efecto debido al anegamiento y arrastre en las laderas. La observación, en el terreno, que la hipotermia causó la muerte de algunos individuos apoya este mecanismo.

El efecto negativo de la abundancia sobre las tasas de maduración sugiere la existencia de mecanismos de competencia intraespecífica por recursos durante la reproducción (Stenseth et al. 1996). En efecto, para otra especie de roedor, Clethrionomys glareolus, cuyas hembras son territoriales (Bujalska 1990), Prévôt-Julliard et al. (1999) determinaron que la probabilidad de maduración de las hembras depende negativamente de la abundancia de las hembras territoriales en una población de Finlandia. Desgraciadamente, como ha sido enfatizado por Lima et al. (2001), no hay datos empíricos sobre la estructura social o territorialidad en $P$. darwini para comprobar o confirmar esta hipótesis. Sin embargo, este resultado está en sintonía con el efecto negativo de la densidad sobre la tasa de crecimiento poblacional durante la estación reproductiva, encontrado por Lima et al. (2002) en la población de $P$. darwini de Aucó.

\section{AGRADECIMIENTOS}

ML agradece el financiamiento de un proyecto FONDECYT 1020513. ML y LC agradecen los financiamientos del proyecto FONDAPFONDECYT 1501-0001 y del proyecto "Demographic and population dynamics modelling of small rodents in semiarid environments" del programa de cooperación científica y tecnológica Chile-comunidad flamenca de Bélgica. Los autores agradecen al profesor Eric Le Boulengé por su lectura crítica de una versión previa del manuscrito.

\section{LITERATURA CITADA}

ARANA M, O RAMÍREZ, S SANTA MARÍA, C KUNIMOTO, R VELARDE, C DE LA CRUZ \& ML RUIZ (2002) Densidad poblacional y reproducción de dos ratones orejudos del Perú. Revista Chilena de Historia Natural 75: 751-756.

BEISSINGER SR \& NFR SNYDER (2002) Water levels affect nest success of the snail kite in Florida: AIC and the omission of relevant candidate models. The Condor 104: 208-215.

BUJALSKA G (1990) Social system of the bank vole Clethrionomys glareolus. En: Tamarin RH, RS Ostfeld, SR Pugh \& G Bujalska (eds) Social systems and population cycles in voles: 155-167. Birkhäuser, Basel, Switzerland.

BURNHAM, KP \& DR ANDERSON (1998) Model selection and inference. A practical information theoretic approach. Springer, Verlag, New York, USA. 353 pp.

CASWELL H (2001) Matrix population models. Construction, analysis, and interpretation. Second edition. Sinauer, Sunderland, Massachusetts, USA. $722 \mathrm{pp}$.

CHOQUET R, AM REBOULET, R PRADEL, O GIMÉNEZ \& JD LEBRETON (2003) User's manual for U-Care. Mimeographed document, CEFE/CNRS, Montpellier (ftp://ftp.cefe.cnrsmop.fr/biom/Soft-CR/).

CHOQUET R, AM REBOULET, R PRADEL, O GIMÉNEZ \& JD LEBRETON (2004) M-SURGE: new software specifically designed for multistates capture-recapture models. Animal Biodiversity and Conservation 27: 207-215.

CRESPIN L, R VERHAGEN, NC STENSETH, NG YOCCOZ, AC PRÉVOT-JULLIARD \& JD LEBRETON (2002) Survival in fluctuating bank vole populations: seasonal and yearly variations. Oikos 98: 467-479.

DREITZ VJ, RE BENNETTS, B TOLAND, WM KITCHENS \& MW COLLOPY (2001) Spatial and temporal variability in nest success of snail kites in Florida: a meta-analysis. The Condor 103: 502509.

DREITZ VJ, RE BENNETTS, B TOLAND, WM KITCHENS \& MW COLLOPY (2002) Snail kite nest success and water levels: a reply to Beissenger and Snyder. The Condor 104: 216-221.

GINZBURG LR \& CXJ JENSEN (2004) Rules of thumb for judging ecological theories. Trends in Ecology and Evolution 19: 216-126.

GROBOIS V \& G TAVECCHIA (2003) A new way of modelling dispersal with capture-recapture data: separating decisions of leaving and settlement. Ecology 84: 1225-1236.

HESTBECK JB, JD NICHOLS \& RA MALECKI (1994) Estimates of movement and sire fidelity using mark-resight data of wintering Canada geese. Ecology 72: 523-533. 
HEPPELL SS, H CASWELL \& LB CROWDER (2000) Life histories and elasticity patterns: perturbation analysis for species with minimal demographic data. Ecology 81: 654-665.

JOHNSON JB \& KS OMLAND (2004) Model selection in ecology and evolution. Trends in Ecology and Evolution 19: 101-108.

JULLIARD R, H LEIRS, NC STENSETH, NG YOCCOZ, AC PRÉVÔT-JULLIARD, R VERHAGEN \& W VERHEYEN (1999) Survival-variation within and between functional categories of the African multimammate rat. Journal of Animal Ecology 68: $550-561$

KANEKO Y, K NAKATA, T SAYITO, NC STENSETH \& ON BJØRNSTAD (1998) The biology of the vole Clethrionomys rufocanus: a review. Researches on population. Ecology 40: 21-37.

KORN H (1989) A feeding experiment with 6methoxybenzoxazolinone and a wild population of the deer mouse (Peromyscus maniculatus). Canadian Journal of Zoology 67: 2220-2224.

KREBS CJ (1966) Demographic changes in fluctuating populations of Microtus californicus. Ecological Monographs 68: 571-594.

LEBRETON JD \& J CLOBERT (1991) Bird population dynamics, management and conservation: the role of mathematical modelling. En: Perrins CM, JD Lebreton \& GJM Hirons (eds) Bird population studies. Relevance to conservation and management: 105-125. Oxford University Press, Oxford, United Kingdom

LEBRETON, JD, KP BURNHAM, J CLOBERT \& DR ANDERSON (1992) Modeling survival and testing biological hypotheses using marked animals: a unified approach with case studies. Ecological Monographs 62: 67-118.

LEIRS H (1994) Population ecology of Mastomys natalensis (Smith, 1834) multimammate rats: possible implications for rodent control in Africa. Agricultural Edition (Bélgica) 35: 5-268

LEIRS H, R VERHAGEN \& W VERHEYEN (1993) Productivity of different generations in a population of Mastomys natalensis rats in Tanzania. Oikos 68 53-60.

LEIRS H, R VERHAGEN \& W VERHEYEN (1994) The basis of reproductive seasonality in Mastomys rats (Rodentia: Muridae) in Tanzania. Journal of Tropical Ecology 10: 55-66.

LEIRS H, NC STENSETH, JD NICHOLS, JE HINES, R VERHAGEN \& W VERHEYEN (1997) Stochastic seasonality and nonlinear density-dependent factors regulate population size in an African rodent. Nature 389: 176-180.

LIMA M \& FM JAKSIC (1998a) Population variability among three small mammal species in the semi-arid Neotropics: the role of density-dependent and density-independent factors. Ecography 21: 175180.

LIMA M \& FM JAKSIC (1998b) Delayed densitydependent and rainfall effects on reproductive parameters of an irruptive rodent in semiarid Chile. Acta Theriologica 43: 225-236.

LIMA M \& FM JAKSIC (1999) Population rate of change in the leaf-eared mouse: the role of densitydependence, seasonality and rainfall. Australian Journal of Ecology 24: 110-116.

LIMA M, JE KEYMER \& FM JAKSIC (1999) ENSOdriven rainfall variability and delayed density dependence cause rodent outbreaks in western South America: linking demography and population dynamics. American Naturalist 153: 476-491.

LIMA M, R JULLIARD, NC STENSETH \& FM JAKSIC (2001) Demographic dynamics of a neotropical small rodent (Phyllotis darwini): feedback structure, predation and climatic factors. Journal of animal Ecology 70: 761-775.

LIMA M, NC STENSETH \& FM JAKSIC (2002) Population dynamics of a South American small rodent: seasonal structure interacting with climate, density-dependence and predator effects. Proceedings of the Royal Society of London B 269: 2579-2586.

LIMA M, NC STENSETH, H LEIRS \& FM JAKSIC (2003) Population dynamics of small mammals in semiarid regions: a comparative study of withinyear demographic variability in two rodent species. Proceedings of the Royal Society of London B 270: 1997-2007.

LINN IJ (1991) Influence of 6-methoxybenzoxazolinone and green vegetation on reproduction of the multimammate rat Mastomys coucha. South African Journal of Wildlife Research 21: 33-37.

MESERVE PL \& E LE BOULENGÉ (1987) Population dynamics and ecology of small mammals in the northern Chilean semi-arid region. Fieldiana Zoology New Series 39: 413-431.

NICHOLS JD, HINES JE, POLLOCK KH, HINZ RL \& WA LINK (1994) Estimating breeding proportions and testing hypotheses about costs of reproduction with capture-recapture data. Ecology 75: 20522065.

NUR N \& WJ SYDEMAN (1999) Demographic processes and population dynamic models of seabirds. Implications for conservation and restoration. Current Ornithology 15: 149-188.

OSTFELD RS \& CD CANHAM (1995) Density-dependent processes in meadow voles: an experimental approach. Ecology 76: 521-532.

OTIS DL, KP BURNHAM, GC WHITE \& DR ANDERSON (1978) Statistical inference from capture data on closed animal populations. Wildlife Monographs 62: 1-135.

PRADEL R, J CLOBERT \& JD LEBRETON (1990) Recent developments for the analysis of capturerecapture multiple data sets. An example concerning two blue tit populations. The Ring (Polonia) 13: 193-204.

PRADEL R, CMA WINTREBERT \& O GIMENEZ (2003) A proposal for a goodness-of-fit test to the Arnason-Schwarz multisite capture-recapture model. Biometrics 59: 43-53

PRÉVÔT-JULLIARD AC, H HENTTONEN, NG YOCCOZ \& NC STENSETH (1999) Delayed maturation in female bank voles: optimal decision or social constraint? Journal of Animal Ecology 68: 684-697.

SINCLAIR JA \& RL LOCHMILLER (2000) The winter immunoenhancement hypothesis: associations among immunity, density, and survival in prairie vole (Microtus ochrogaster) populations. Canadian Journal of Zoology 78: 254-264.

STENSETH NC, BJØRNSTAD ON \& FALCK W (1996) Is spacing behaviour coupled with predation causing the microtine density cycle? A synthesis of current process-oriented and pattern-oriented studies. Proceedings of the Royal Society of London B 263: 1423-1435.

WHITE GC \& KP BURNHAM (1999) Program MARK: survival estimation from populations of marked animals. Bird Study (Reino Unido) 46: 120-139. 
YOCCOZ NG \& S MESNAGER (1998) Are alpine bank voles larger and more sexually dimorphic because adults survive better? Oikos 82: 85-98.

YOCCOZ NG, K NAKATA, NC STENSETH \& $T$

Editor Asociado: Rodrigo Ramos-Jiliberto

Recibido el 8 de febrero de 2006; aceptado el 27 de junio de 2006
SAITOH (1998) The demography of Clethrionomys rufocanus: from mathematical and statistical models to further field studies. Research Population Ecology 40:107-121. 